\title{
Social consensus and tipping points with opinion inertia
}

\author{
C. Doyle ${ }^{1,2}$, S. Sreenivasan ${ }^{1,2,3}$, B. K. Szymanski ${ }^{2,3}$, G. Korniss ${ }^{1,2 *}$ \\ 1 Dept. of Physics, Applied Physics and Astronomy, Rensselaer Polytechnic Institute 110 \\ Eighth Street, Troy, NY 12180 \\ ${ }^{2}$ Network Science and Technology Center, Rensselaer Polytechnic Institute 110 Eighth \\ Street, Troy, NY 12180 \\ 3 Dept. of Computer Science, Rensselaer Polytechnic Institute 110 Eighth Street, Troy, NY \\ 12180
}

\begin{abstract}
When opinions, behaviors or ideas diffuse within a population, some are invariably more sticky than others. The stickier the opinion, behavior or idea, the greater is an individual's inertia to replace it with an alternative. Here we study the effect of stickiness of opinions in a two-opinion model, where individuals change their opinion only after a certain number of consecutive encounters with the alternative opinion. Assuming that one opinion has a fixed stickiness, we investigate how the critical size of the competing opinion required to tip over the entire population varies as a function of the competing opinion's stickiness. We analyze this scenario for the case of a complete-graph topology through simulations, and through a semi-analytical approach which yields an upper bound for the critical minority size. We present analogous simulation results for the case of the Erdős-Rényi random network. Finally, we investigate the coarsening properties of sticky opinion spreading on two-dimensional lattices, and show that the presence of stickiness gives rise to an effective surface tension that causes the coarsening behavior to become curvature-driven.
\end{abstract}

Keywords: opinion dynamics, social networks, influencing, tipping points, opinion inertia

\section{Introduction}

Social networks represent potent structures on which opinions and behavior diffuse, and on which tipping points [1] in the adoption of opinions and behavior arise. A number of theoretical studies investigating the diffusion of ideas, opinions, or behavior, have focussed on understanding how a small fraction of initiators $[2,3,4]$ or committed proselytizers $[5,6,7,8,9,10]$ of an idea can tip

\footnotetext{
* Corresponding author

Email address: korniss@rpi.edu (G. Korniss ${ }^{1,2}$ )
}

Preprint submitted to Physica A

June 29, 2015 
over the entire network to adopt the same. Furthermore, within these studies, various sources of competition to the spread of an idea have been considered - for example, a competing idea that is spreading over the network, or a bias external to the network that is trying to suppress the spread of an idea. More pertinently, however, the tendency of individuals themselves to be pliable to change is dynamic and could be dependent on their activity. In particular, individual behavior itself is subject to some inertia that opposes any change in the beliefs or opinions adopted by the individual [11]. A well known example of such inertia is the phenomenon of confirmation bias in social psychology, where individuals tend to favor beliefs that conform to their currently held position. Overcoming such individual inertia to change is therefore a primary consideration in campaigns for public opinion change [12].

Motivated by this phenomenon, we study a theoretical model of opinion change where individual opinion change depends on the current state of the individual as well as the recent history of the opinions she has encountered in interactions with her neighborhood. Specifically, we assume that there are two opinions vying for adoption on a social network, and each individual requires a pre-defined threshold number of interactions with the alternative opinion, before switching to it. Thus each opinion is sticky to its respective extent [12]. Furthermore, in an attempt to capture the effect of confirmation bias, we posit that an individual's memory of a stream of encounters with the alternative opinion is erased by a single interaction in which he encounters his currently held opinion. There is some precedent to studying such a memory-based model of switching between states. Dodds and Watts [13] studied a model of disease contagion where a susceptible person became infected only when his interactions with infected neighbors within a certain prior time window had led to a pre-defined infection-dosage threshold being exceeded. More pertinently to the current study, Dall'Asta and Castellano [14] studied a variant of the Naming Game with two pure opinions, where an individual switches to the intermediate state only when the number of times he has encountered the opposing opinion exceeds some pre-defined threshold. Our model thus is a special case of [14] where the memory window is exactly equal to the threshold, and where no intermediate state is present. In contrast to the work done in [14], here our focus is to look at the fraction of initiators required to bring about a tipping point. The effect of stickiness has also been studied in the context of the Naming Game in [15] and more recently in [16]. In these studies, the stickiness parameter quantifies the probabilities with which a node in a mixed-opinion state rejects a pure state that it encounters in an interaction with its neighbors. The introduction of the stickiness parameter for nodes in the mixed-opinion state, gives rise to a phase transition between a regime where the consensus states are stable (when stickiness is low) to one where the consensus states are unstable and the system gravitates to a stable state with a non-zero density of mixed-opinion nodes.

A recent work [17] has studied a variant of the SIR model where the infection probability is a function of the number of infectious neighbors, and parametrized by two parameters that they designate as stickiness and persistence. Despite the nomenclature, the term stickiness is utilized in [17] to designate the slope 
of the infection probability of a susceptible node as a function of the size of its infected neighborhood, and therefore bears little similarity to the context that we study. Finally, recent empirical findings [18] demonstrate the dependence of social network properties on cultural attributes of the population, suggesting that stickiness could also be similarly influenced by cultural factors.

\section{Description of model}

Here we define the microscopic rules of our model. We assume that every individual on a social network initially adopts one of two opinions, which we designate $A$ and $B$. The fundamental mechanism in our model for the change in individual states is the interaction of pairs of individuals, which represent speaker-listener pairs. In each such interaction, the speaker conveys his opinion to the listener, and in response to this conveyed opinion, the listener changes his state or continues to hold the same state depending on the rules of the model. We elaborate on these rules in the next few lines. First, each opinion has a predefined stickiness, designated as $w_{A}$ and $w_{B}$ respectively. The stickiness of an opinion represents the inertia present in an individual adopting that opinion, to change her state. In terms of the model, the stickiness $w_{A}\left(w_{B}\right)$ of an individual in state $A(B)$ is the number of consecutive times she requires to hear the opinion $B(A)$, before she switches her opinion to $B(A)$. Thus, we can assume that each individual keeps a counter dedicated to counting the number of times she encounters the alternative opinion, which resets to zero either when the required number of consecutive interactions of the alternative opinion are heard, or whenever the current opinion is heard. Note that in the former case, the counter also switches the opinion that it is keeping track of. In our current model implementation, we assumed that exposure to a different/same opinion only impacts the individuals' counter when their role is the listener in a pairwise interaction. Naturally, one may consider the scenario where both the speaker's and listener's counters are affected by the interactions (i.e., the speaker can also be reinforced in her view). We did some explorations on this generalization of the model, and have found that there are no qualitative differences in the results.

In summary, the model dynamics proceeds as follows. The individuals (nodes) in the network are initially assigned one of the two opinions such that we have prescribed fractions $p_{A}$ and $p_{B}=1-p_{A}$ of nodes in states $A$ and $B$ respectively. Then at each microscopic time step, a random node is chosen from the system and designated as the speaker. A random node is selected from among the speaker's neighbors and designated as the listener. If the listener's opinion is the same as the speaker's, it's progress towards switching is reset to zero. If the listener's opinion is different from the speaker's, the listener's count towards switching increases by one. If the listener's count becomes equal to it's opinion's stickiness, it adopts the alternative opinion and begins a fresh count. We assume that $N$ such microscopic time steps constitute unit time, where $N$ is the network size. Thus, the event that a node is selected as a speaker is a Poisson process with rate 1. 


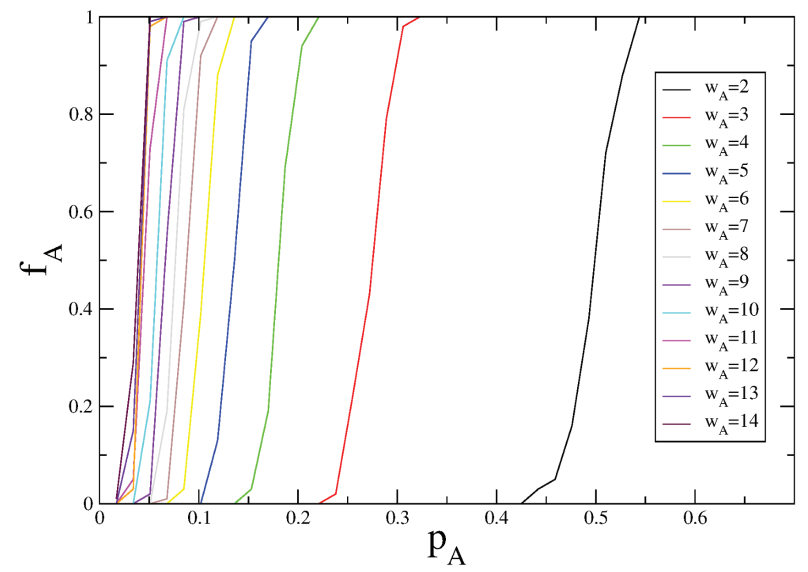

Figure 1: The fraction of simulation runs on a complete graph with $N=1000$ that reach consensus on opinion $A$ vs. the initial population fraction of opinion $A$ for different stickiness values of the $A$ opinion. For these simulations $w_{B}=2$.

\section{Results and Discussion}

\subsection{Complete Graph}

First, we investigate the outcome of these rules on a complete graph through Monte-Carlo simulations. As shown in Fig. 1, we vary the fraction, $p_{A}$, of nodes adopting opinion $A$, and measure the fraction of simulation runs (over a total of 500 runs) for which the system reaches consensus on opinion $A$. We keep the stickiness of the opinion $B$ fixed at $w_{B}=2$ and vary the stickiness $w_{A}$ of opinion $A$. For a finite system, for every value of $w_{A}$, the fraction of runs reaching consensus on opinion $A, f_{A}$, follows a typical S-shaped curve [Fig. 1]. For increasing system sizes, these curves are becoming progressively sharper [Fig. 2(a)], approaching a discontinuous transition in the infinite system-size limit and indicating the existence of a tipping point at a critical fraction $p_{c}$. For a finite system size $N$, we identify $p_{c}$ where the (forward) derivative of the fraction of runs reaching $A$-consensus, $\chi \equiv d f_{A} / d p_{A}$, is maximum [Fig. 2(b)]. These results also indicate that the finite-size effects of the location of the critical point are negligible for this transition.

As demonstrated by the results shown in Fig. 1, for values of $w_{A} \geq 3$, at the critical point, the opinion $A$ initially constitutes the minority opinion. Thus, having a stickiness even marginally greater than that of the majority opinion allows the minority opinion to tip over the entire population, as long as the minority fraction is greater than $p_{c}$. For equal stickiness $w_{A}=w_{B}=2$, the fraction of opinion $A$ holders must from the start be the majority opinion, in order to win over the population.

As the stickiness of the minority opinion is increased, the takeover of the entire network occurs at progressively smaller minority fractions. As shown in Fig. $3, p_{c}$ appears to converge to zero as $w_{A} \rightarrow \infty$. For simulations shown here, 
(a)

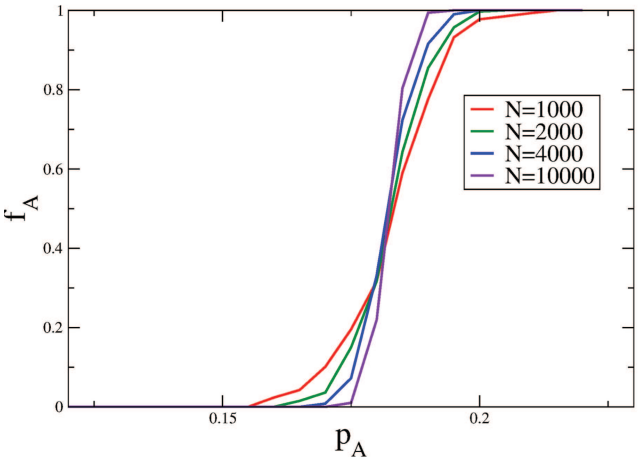

(b)

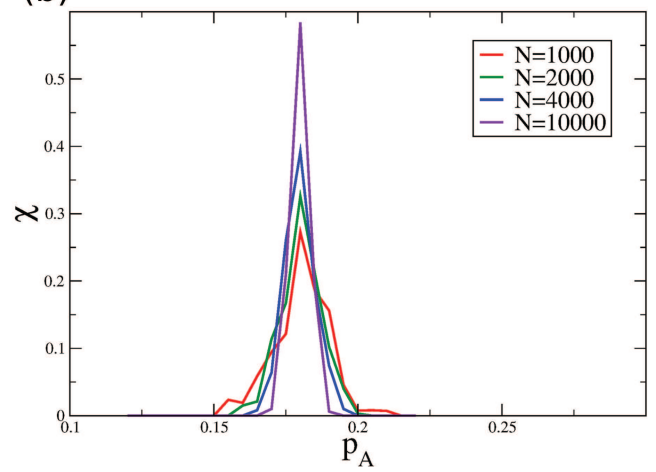

Figure 2: (a) The fraction of runs reaching consensus on opinion $A$ vs. the initial population fraction of opinion $A$ for different system sizes. These runs have $w_{A}=4, w_{B}=2$, and are averaged over 1000 runs. (b) Forward derivative of the fraction of runs reaching consensus on opinion $A$ from (a) vs. the initial population fraction of opinion $A$.

$N=1000$, and hence the smallest value that $p_{c}$ can adopt is 0.001 . However, we show using a semi-analytic approach (Sec. 3.2) that an upper bound to the critical value $p_{c}$ itself converges to 0 as $w_{A} \rightarrow \infty$ [Fig. 3(a) inset], which confirms that the critical fraction vanishes for asymptotically large stickiness.

Note that the full dependence of the tipping point $p_{c}$ on the stickiness parameters $w_{A}$ and $w_{B}$ is rather complex and non-linear. Our simulation results and scaling suggest that

$$
p_{c} \simeq \frac{\text { const. }}{\left(w_{A}^{1 / 2} / w_{B}\right)} \propto w_{B} w_{A}^{-1 / 2},
$$

in the $1 \ll w_{B} \ll w_{A}$ limit, as shown in the inset of Fig. 3(b).

\subsection{Analytical approximation for critical fraction on complete graphs}

For convenience, in this subsection we denote the stickiness of opinion $A$ by $w$ and the stickiness of opinion $B$ by $v$. We denote the fraction of nodes holding opinions $A$ and $B$ by $n_{A}$ and $n_{B}$ respectively. The fraction of nodes holding opinion $A$ is comprised of distinct subpopulations that hold opinion $A$ and have accrued a certain number of consecutive hits from opinion $B$. We denote the fractional sizes of these subpopulations by $s_{a, 0}, s_{a, 1}, \cdots, s_{a, w-1}$ respectively. Analogously, the subpopulations for opinion $B$ are denoted as $s_{b, 0}, s_{b, 1}, \cdots, s_{b, v-1}$ respectively. Thus

$$
\begin{aligned}
& n_{A}=\sum_{x=0}^{x=w-1} s_{a, x} \\
& n_{B}=\sum_{x=0}^{x=v-1} s_{b, x} .
\end{aligned}
$$


(a)

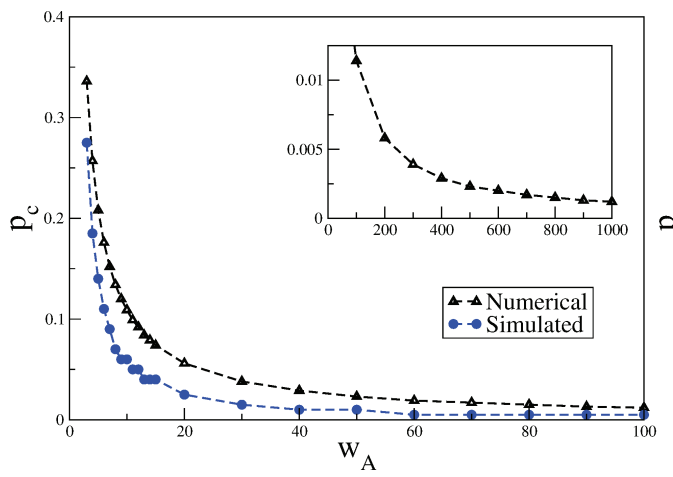

(b)

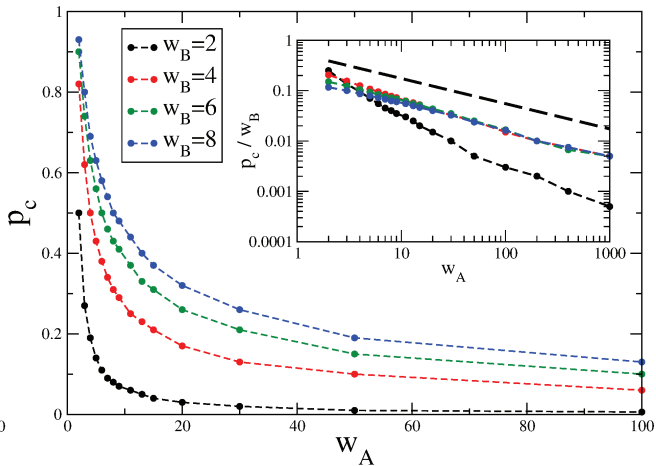

Figure 3: (a) Comparison between the values of the critical fraction $p_{c}$ as a function of the stickiness of opinion A for a complete-graph with $N=1000$ obtained from simulations (averaged over 100 runs) and through the semi-analytic approach (Sec. 3.2). In both cases $w_{B}=2$. The inset shows the extended numerical results [using Eqs. (6) and (8) up to values of $w_{A}=1000$. (b) Critical populations obtained by simulations for different values of $w_{B}$ with $N=1000$ and averaged over 100 runs. The inset shows the scaled data on a log-log scale. The dashed line, for reference, corresponds to a power law with exponent $-1 / 2$.

We can write evolution equations for the density of nodes in states $A$ and $B$, by noting that a change in opinion occurs when a node whose counter for the alternate opinion is just below the stickiness of its current opinion, encounters the alternate opinion. Thus, the density of nodes with opinion $A$ evolves according to the equation:

$$
\frac{d n_{A}}{d t}=-n_{B} s_{a, w-1}+n_{A} s_{b, v-1},
$$

where the first term captures the loss of nodes in state $A$, resulting from nodes represented by the fraction $s_{a, w-1}$ hearing opinion $B$. The second term analogously captures the gain resulting from nodes represented by the fraction $s_{b, v-1}$ hearing opinion $A$. Similarly,

$$
\frac{d n_{B}}{d t}=-n_{A} s_{b, v-1}+n_{B} s_{a, w-1},
$$

Next, in order to make these equations tractable, we introduce a quasi-steady state approximation for obtaining the subpopulation fractions for each opinion. Specifically, we assume:

$$
\begin{aligned}
& s_{a, x}=s_{a, 0}\left(n_{B}\right)^{x} \\
& s_{b, x}=s_{b, 0}\left(n_{A}\right)^{x} .
\end{aligned}
$$

Namely, we assume that the fraction of nodes in state $\{a, x\}$ at a given time is approximately equal to the probability, given the systems current state, 
of picking a node in state $\{a, 0\}$, and picking a node in state $B$ on every one of $x$ trials with replacement. This assumption, commonly used in the study of chemical reaction systems with intermediates, is known as the quasi-steadystate assumption [19], referring to the fact that the intermediate subpopulations arising in the transition from state $\{a, 0\}$ to state $\{b, 0\}$ and vice-versa, are assumed to be in steady-state. This can be seen from the evolution equation for a particular subpopulation, say $\{a, x\}$ :

$$
\frac{d s_{a, x}}{d t}=-n_{A} s_{a, x}-n_{B} s_{a, x}+n_{B} s_{a, x-1}
$$

Since $n_{A}+n_{B}=1$, the steady-state expression for fraction of nodes in state $\{a, x\}$ is: $s_{a, x}=n_{B} s_{a, x-1}$. Thus, $s_{a, x}=\left(n_{B}\right)^{x} s_{a, 0}$. Using this approximation, Eqs. (3), (4) become:

$$
\begin{aligned}
\frac{d n_{A}}{d t} & =-\left(n_{B}\right)^{w} s_{a, 0}+\left(n_{A}\right)^{v} s_{b, 0} \\
\frac{d n_{B}}{d t} & =-\left(n_{A}\right)^{v} s_{b, 0}+\left(n_{B}\right)^{w} s_{a, 0}
\end{aligned}
$$

Additionally, we have evolution equations for nodes in states $\{a, 0\}$ and $\{b, 0\}$ as well:

$$
\begin{aligned}
& \frac{d s_{a, 0}}{d t}=-n_{B} s_{a, 0}+n_{A} \sum_{x=1}^{w-1} s_{a, x}+n_{A} s_{b, v-1} \\
& \frac{d s_{b, 0}}{d t}=-n_{A} s_{b, 0}+n_{B} \sum_{x=1}^{v-1} s_{b, x}+n_{B} s_{a, w-1}
\end{aligned}
$$

and using the quasi-steady-state assumption and Eqs. (2), we obtain:

$$
\begin{aligned}
& \frac{d s_{a, 0}}{d t}=-n_{B} s_{a, 0}+n_{A}\left(n_{A}-s_{a, 0}\right)+\left(n_{A}\right)^{v} s_{b, 0} \\
& \frac{d s_{b, 0}}{d t}=-n_{A} s_{b, 0}+n_{B}\left(n_{B}-s_{b, 0}\right)+\left(n_{B}\right)^{w} s_{a, 0}
\end{aligned}
$$

We numerically solve the coupled equations, Eqs. (6) and (8) for different initial fractions $n_{A}^{\text {init }}, n_{B}^{\text {init }}$ (with $n_{A}^{\text {init }}<n_{B}^{\text {init }}$ ) and obtain the steady state values of $n_{A}$ and $n_{B}$ respectively. We then record the smallest value of $n_{A}^{\text {init }}$ at which the steady state value of $n_{A}$ becomes greater than 0.99 and designate this as the critical initial minority fraction $p_{c}$ required to tip the system over. Figure 3 shows a comparison for the tipping point $p_{c}$ obtained through this semi-analytical approach and that obtained from simulation for different stickiness values of opinion $A$ (while the stickiness of the other opinion is held fixed at $w_{B}=2$ ). The cause of the higher $p_{c}$ values yielded by the semi-analytical approach is the overestimation of subspecies densities $\left(s_{a, x}, s_{b, x}\right.$ for $\left.x>0\right)$ in the initial phase of the dynamics - in reality the subspecies densities take some length of time to attain non-zero values. This overestimation favors the sustenance of nodes in state $B$, 


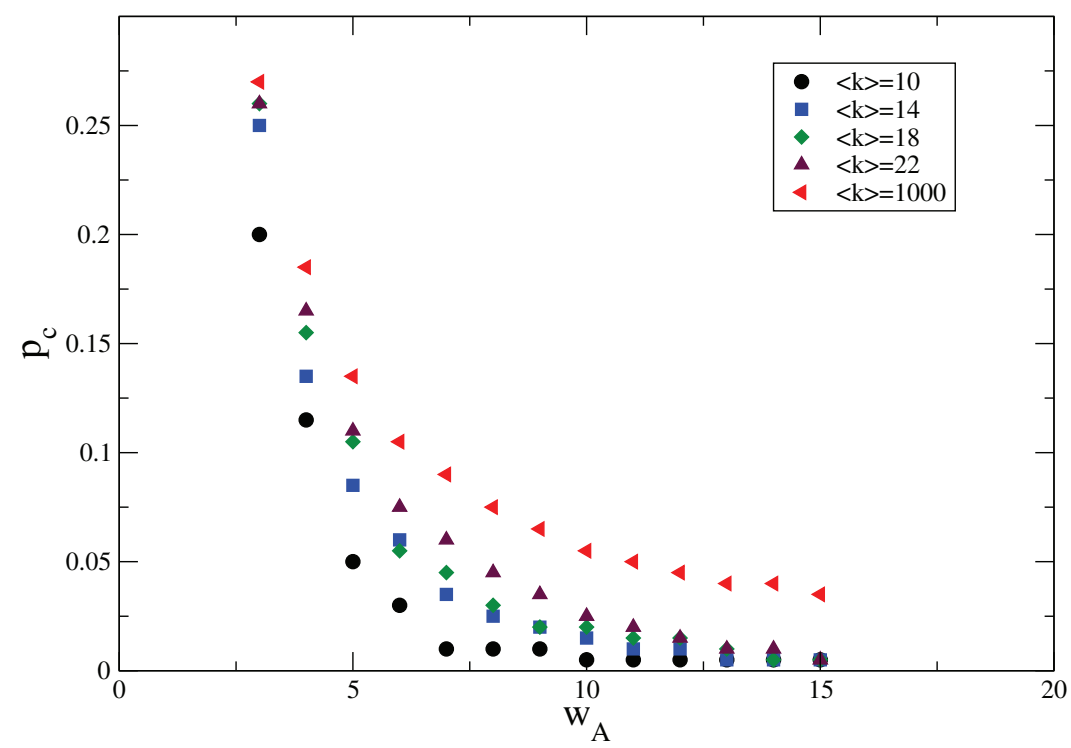

Figure 4: The critical fraction $p_{c}$ vs. the stickiness of opinion A on Erdős-Rényi graphs with $N=1000$ for various average degree $\langle k\rangle$.

since they are initially in the majority. As a result, the fraction of nodes in state $A$ required to tip the system over, as estimated by the quasi-steady-state approximation, is larger. Thus, the semi-analytical estimate of $p_{c}$ consistently represents an upper-bound to the value observed in simulations. Furthermore, in the event that the stickiness of opinion $A$ diverges, Eq. (6) shows that for any non-zero initial density of $A$ opinions, $n_{A}$ grows monotonically while $n_{B}$ decays monotonically, showing that the true critical fraction $p_{c}$ is bounded above by a value that vanishes in the asymptotic limit of stickiness.

\subsection{Erdös-Rényi random graph}

A similar asymptotic dependence of $p_{c}$ on $w_{A}$ with $w_{B}=2$ is observed for Erdős-Rényi random graphs of size $N=1000$, as shown in 4 . Lowering the average degree of the graph $\langle k\rangle$ tends to lower the critical value. For comparison, we also show the critical values obtained for the corresponding complete graph with 1000 nodes.

\subsection{Curvature-driven coarsening in the presence of stickiness}

Next, we investigate how the introduction of stickiness into the rules of opinion change affects the coarsening behavior of the system. To facilitate comparison with previous studies, we investigate the evolution of a circular droplet of nodes in state $A$ immersed in a sea of nodes holding opinion $B$ in two dimensions. First, we visually inspect this evolution under the rules of the model, for various combinations of values for $w_{A}$ and $w_{B}$. The nodes in this 
case, are the sites of a square lattice (with each node connected to 4 nearest neighbors) of side $L=250$ without periodic boundary conditions. The droplet initially has a radius of $R_{0}=35$. For $w_{A}=w_{B}=1$, there is no stickiness in either opinion, and the dynamics reduces to that of the voter model [20], where one interaction with the alternative opinion is sufficient to cause a node to change its opinion. Figure 5(a) shows the evolution of the droplet in this case. As demonstrated in a previous study [21], the noise-driven roughening of the interface is clearly visible as the droplet evolves. Next, we introduce stickiness in the opinions by assuming $w_{A}=w_{B}=2$. The initial conditions are identical to those for the case shown in Fig. 5(a). Figure 5(b) shows a markedly different picture and the presence of an effective surface tension in the model is evident from the preservation of interface smoothness over time. This curvature-driven evolution is consistent with behavior observed in prior studies on voter-like models with intermediate states [22, 23, 24] or memory [14], since the effect of stickiness (or memory) is similar to that of intermediate states that intercede the transition between two opinions. Finally, stickiness in only one of the two opinions is sufficient (see Figs. 5(c),(d)) to keep curvature-driven behavior intact.

Next, we investigate the coarsening behavior quantitatively. We track the evolution of the density of interfaces, $\rho(t)$ i.e. the fraction of nearest-neighbor pairs which differ in their opinion. This is a commonly used order parameter that characterizes the coarsening behavior [25, 26]. For curvature-driven coarsening systems, the radius of the droplet changes linearly with time [27]. In $2 \mathrm{D}$, it follows that the interface density also grows or decays linearly in time i.e. $\rho(t) \sim$ $c_{1} \pm c_{2} t$, where $c_{1}$ and $c_{2}$ are constants. Whether the droplet grows or decays depends on both the initial size of the droplet, as well as the values of stickiness for the two opinions. As shown in Fig. 6(a), the decay in interface density is indeed linear, as predicted by theory. Here, the initial radius of the droplet is $R=35$, the lattice size is $L=250$, and the stickiness parameters are $w_{A}=$ $w_{B}=2$. Figure $6(\mathrm{~b})$ shows the fraction of simulation runs (over a total of 400 runs) for which the droplet grows and spreads over the entire lattice (with $L=35$ ) as a function of the initial droplet radius for various combinations of stickiness. The results indicate the existence of a critical initial droplet radius for every combination, such that the probability of droplet growth sharply rises for initial radii above this critical value [27].

In diffusive systems like the voter model, it has been theoretically demonstrated that in the asymptotic long-time limit, the interface density decays logarithmically [28] in 2D, under random initial conditions (see details below). Figure 7(a) shows two snapshots of the coarsening process for the case $w_{A}=w_{B}=1$, on a $100 \times 1002 \mathrm{D}$ square lattice at time $t=0$ (random initial conditions) and at $t=25$, respectively. The diffusive nature of interface evolution, characteristic of the voter model, is clearly visible and is consistent with the behavior observed in the evolution of the circular droplet shown in Figure 5(a).

Figure $7(\mathrm{~b})$ shows the slow decay of the interface density as a function of time. One must be careful, however, as the exact asymptotic inverse log- 
(a)

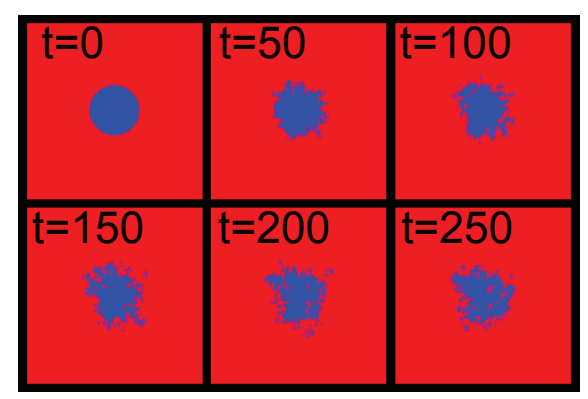

(c)

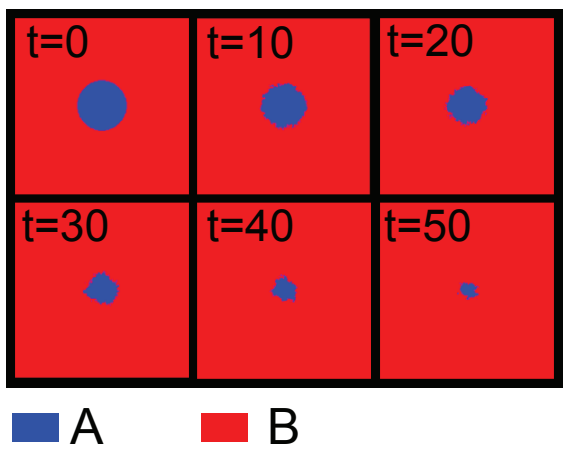

(b)

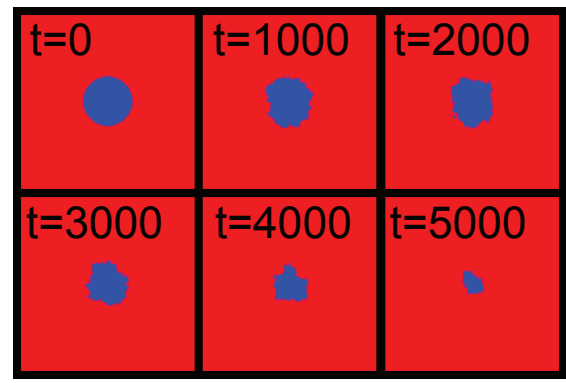

(d)

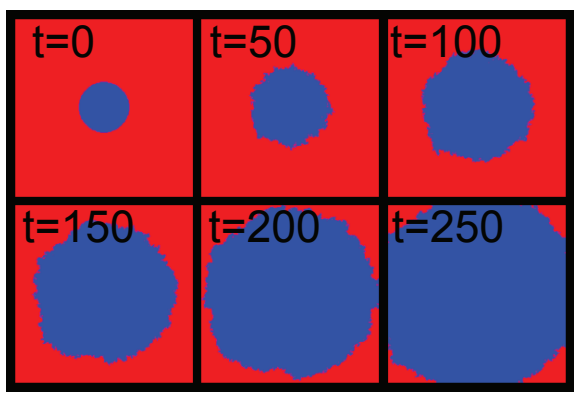

Figure 5: Snapshots of the evolution of a droplet of opinion $A$ nodes in a sea of $B$ nodes under different combinations of stickiness parameters. The nodes occupy the sites of a $250 \times 2502 \mathrm{D}$ square lattice without periodic boundaries. Opinion $A$ is in the minority in every case and represented in blue. Nodes with opinion $B$ are colored red. (a) Without stickiness i.e. $w_{A}=$ $w_{B}=1$, the model becomes identical to the voter model, and consistent with observations for the latter, the interface roughens diffusively, without any perceivable surface tension. With the introduction of stickiness in at least one of the two opinions, (b) $w_{A}=w_{B}=2$, (c) $w_{A}=1, w_{B}=2$, (d) $w_{A}=2, w_{B}=1$, the interface evolution becomes curvature driven, and the droplet retains its roughly circular shape as it grows or decays.

arithmic dependence of the interface density on time has long been known to be challenging to demonstrate numerically $[28,29]$. Specifically, for the voter model, the leading-order asymptotic behavior for the interface density is $\rho \simeq \pi /[2 \ln (t)+\ln (256)][28]$. As indicated by the results of our simulations [Fig. 7(c)], our model with $w_{A}=w_{B}=1$ approaches (albeit slowly) precisely this type of long-time asymptotic behavior, as expected.

\section{Conclusions}

We have modeled a scenario where two competing opinions, ideas or behaviors vie for adoption in a social network. Each opinion is endowed with 
(a)

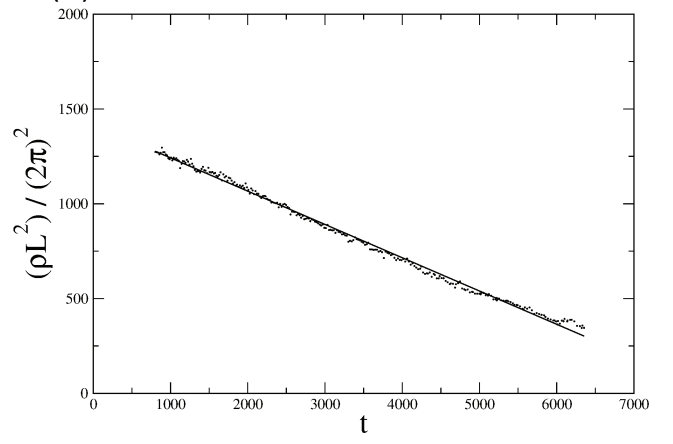

(b)

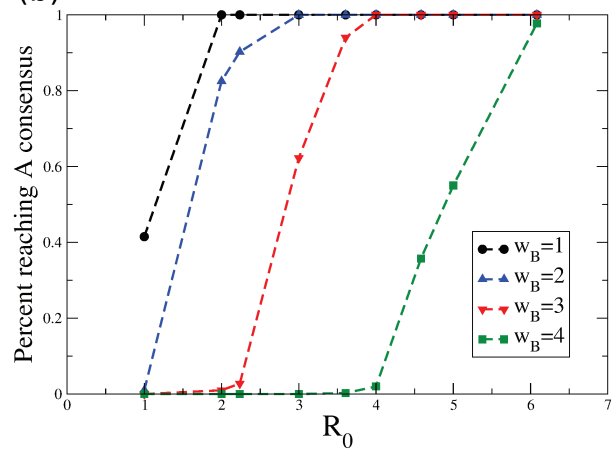

Figure 6: (a) The radius of a circular droplet of opinion $A$ nodes in a sea of $B$ of nodes as a function of time for $w_{A}=w_{B}=2$. The radius is expressed in terms of the interface density $\rho$ and the lattice size (linear dimension of the $2 \mathrm{D}$ square lattice) $L=250$, and shows a linear decrease with time. (b) The growth or decay of the circular droplet depends on its initial radius. Shown here is the fraction of simulation runs where the initial droplet grows and takes over all sites on the lattice. In these simulations, the stickiness for opinion $A$ is held fixed at $w_{A}=5$ and the square lattice has dimensions $35 \times 35$.

an inherent stickiness that impedes an individual adopting that opinion from switching to the alternative opinion.

We have demonstrated that the stickiness of the dominant opinion on a social network determines how large the fraction of minority opinion holders needs to be in order to tip over the population to the initially minority opinion. We have further shown that increasing the stickiness of the minority opinion lowers the critical fraction required for its mass adoption dramatically as shown in Fig. 3. In practical contexts, the stickiness of an opinion or behavior is related to the costs incurred, or incentives provided by its adoption, in comparison with the alternative. On two-dimensional lattices, we have shown that the presence of stickiness in just one of the two opinions causes the system's behavior to belong to the universality class of models where coarsening is curvature-driven. In contrast, in the absence of stickiness, the system belongs to the universality class of the voter model, where coarsening is noise-driven.

In future work, it would be worthwhile investigating the relationship between the ratio of the stickiness values, and the critical value corresponding to the tipping point. Furthermore, empirical data from venues like massively multiplayer online role playing games [30] could be used as a test bed for validating our model and estimating the parameters which govern inertial in opinion change. Lastly, controlled experiments with incentives on online labor markets [31] could further narrow down the conditions under which stickiness becomes a discernible feature of opinion dynamics. 
(a)

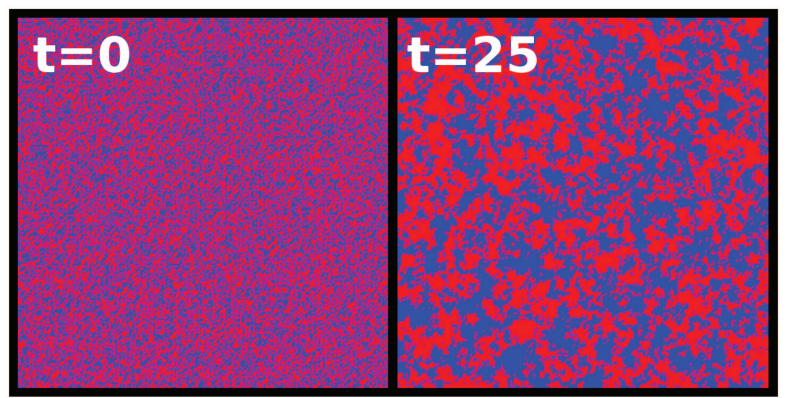

(b)

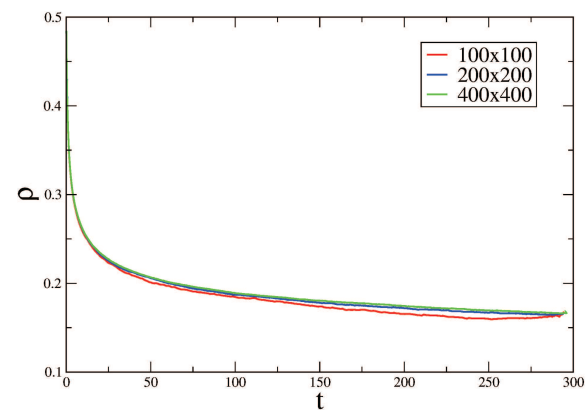

(c)

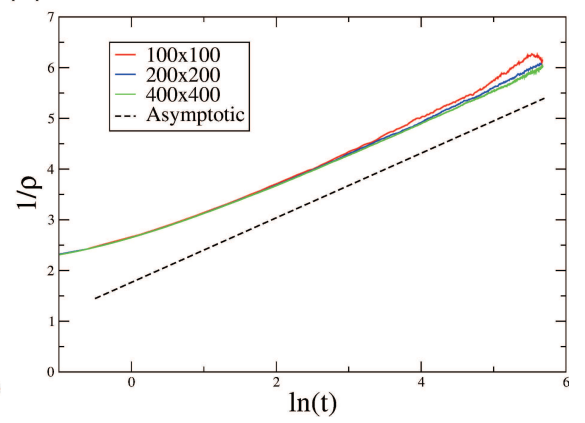

Figure 7: (a) Snapshots of the evolution of a system from random initial condition for $w_{A}=$ $w_{B}=1$ (becoming equivalent to the voter model). The color code is the same as in Fig. 5 . The lattice is a $100 \times 1002 \mathrm{D}$ square lattice with open boundary conditions. (b) The interface density $\rho$ as a function of time $t$ on a $2 \mathrm{D}$ lattice with $w_{A}=w_{B}=1$ for various system sizes. (c) The same simulation data as in (b) but plotted as the inverse interface density vs logarithmic time in order to compare to the exact asymptotic limit of the voter model, $1 / \rho \simeq(2 / \pi) \ln (t)+\ln (256) / \pi$ (dashed line) $[28]$.

\section{Acknowledgments}

This work was supported in part by the Army Research Laboratory (ARL) under Cooperative Agreement Number W911NF-09-2-0053, by the Army Research Office (ARO) grant W911NF-12-1-0546, by the Office of Naval Research (ONR) Grant No. N00014-09-1-0607, and by the National Science Foundation (NSF) Grant No. DMR-1246958. The views and conclusions contained in this document are those of the authors and should not be interpreted as representing the official policies either expressed or implied of the Army Research Laboratory or the U.S. Government. 


\section{References}

[1] M. Gladwell, The Tipping Point: How Little Things Can Make a Big Difference, Back Bay Books, 2002.

[2] D.J. Watts, Proc. Natl. Acad. Sci. USA 99 (2002) 5766-5771.

[3] D.J. Watts and P.S. Dodds, Journal of Consumer Research 34 (2007) 441458.

[4] P. Singh, S. Sreenivasan, B.K. Szymanski and G. Korniss, Sci. Rep. 3 (2013) 2330 .

[5] S. Galam and F. Jacobs, Physica A 381 (2007) 366-376.

[6] J. Xie, S. Sreenivasan,G. Korniss, W. Zhang, C. Lim and B.K. Szymanski, Phys. Rev. E 84 (2011) 011130.

[7] W. Zhang, C. Lim, S. Sreenivasan, J. Xie, B.K. Szymanski, G. Korniss, Chaos 21 (2011) 025115.

[8] J. Xie, J. Emenheiser, M. Kirby, S. Sreenivasan, B.K. Szymanski, G. Korniss, PLoS One 7 (2012) e33215.

[9] M. Turalska, B.J. West, and P. Grigolini, Sci. Rep. 3 (2013) 1371.

[10] A. Waagen, G. Verma, K. Chan, A. Swami, and Raissa D'Souza, Phys. Rev. E 91 (2015) 022811.

[11] R.S. Nickerson, Review of General Psychology 2 (1998) 175-220.

[12] C. Heath and D. Heath, Made to Stick: Why Some Ideas Survive and Others Die, Random House, 2007.

[13] P. Dodds and D. Watts, J. Theor. Biol. 232 (2005) 587-604.

[14] L. Dall'Asta and C. Castellano, Europhys. Lett. 77 (2007) 60005.

[15] A. Baronchelli, L. Dall'Asta, A. Barrat and V. Loreto, Phys. Rev. E 76 (2007) 051102.

[16] A.M. Thompson, B.K. Szymanski and C. Lim, Phys. Rev. E 90 (2014) 042809 .

[17] P. Cui, M. Tang and Z. Wu, Sci. Rep. 4 (2014) 6303.

[18] D. Galehouse, T. Nguyen, S. Sreenivasan, M. Lizardo, G. Korniss and B.K. Szymanski, Impact of network connectivity and agent commitment on spread of opinions in social networks, Proc. AHFE 2014 (2014) 2318.

[19] G.E. Briggs and J.B. Haldane, Biochem. J. 19 (1925) 338. 
[20] T.M. Liggett, Stochastic Interacting Systems: Contact, Voter and Exclusion Processes, Springer-Verlag, 1999.

[21] I. Dornic, H. Chaté, J. Chave and H. Hinrichsen, Phys. Rev. Lett. 87 (2001) 045701.

[22] L. Dall'Asta and T. Galla, J. Phys. A: Mathematical and Theoretical 41 (2008) 435003.

[23] F. Vazquez and C. Lopez, Phys. Rev. E 78 (2008) 061127.

[24] W. Zhang, C. Lim, G. Korniss and B.K. Szymanski, Sci. Rep. 4 (2014) 5568.

[25] C. Castellano, D. Vilone and A. Vespignani, Europhys. Lett. 63 (2003) 153-158.

[26] C. Castellano, V. Loreto, A. Barrat, F. Cecconi and D. Parisi, Phys. Rev. E $71(2005) 066107$.

[27] A. Bray, Advances in Physics 43 (1994) 357-459.

[28] L. Frachebourg and P.L. Krapivsky, Phys. Rev. E 53 (1996) R3009-R3012.

[29] J.W. Evans and T.R. Ray, Phys. Rev. E 47 (1993) 1018-1025.

[30] A. Grabowski, Physica A 388 (2009) 961-966.

[31] S. Suri and D.J. Watts, PLoS ONE 6 (2011) e16836. 